\title{
ON THE BOUNDARY VALUES OF THE SOLUTIONS OF LINEAR ELLIPTIC EQUATIONS
}

\author{
J. Chabrowski and H.B. Thompson
}

\begin{abstract}
The purpose of this article is to investigate the traces of weak solutions of a linear elliptic equation. In particular, we obtain a sufficient condition for a solution belonging to the Sobolev space $W_{10 c}^{1,2}$ to have an $L^{2}$-trace on the boundary.
\end{abstract}

\section{Introduction}

This paper deals with $L^{2}$-behaviour near the boundary of weak solutions of the elliptic equation

$$
L u=-\sum_{i, j=1}^{n} D_{j}\left(a_{i j}(x) D_{i} u\right)+\sum_{i=1}^{n} b_{i}(x) D_{i} u+c(x) u=f(x)
$$

in a bounded domain $Q$. The problem we are concerned with originates in the theory of analytic functions. We say that the analytic function $f(z)$ defined on $(|z|<1)$ has a limit in $L^{2}$ on the boundary, if there exists a function $\phi \in L^{2}(0,2 \pi)$ such that

$$
\lim _{r \rightarrow 1-0} \int_{0}^{2 \pi}\left|f\left(r e^{i \theta}\right)-\phi(\theta)\right|^{2} d \theta=0
$$

Riesz [14] proved the following criterion: the analytic function $f(z)$ on $(|z|<1)$ has a limit in $L^{2}$ on the boundary if and only if the function

Received 20 May 1982. 


$$
U(r)=\int_{0}^{2 \pi}\left|f\left(r e^{i \theta}\right)\right|^{2} d \theta, 0 \leq r<1,
$$

is bounded. Later on Littlewood and Paley [6] established the following theorem: the analytic function $f(z)$ on $(|z|<1)$ has a limit in $L^{2}$ on the boundary if and only if the function

$$
g(\theta)=\left[\int_{0}^{1}(1-r)\left|f^{\prime}\left(r e^{i \theta}\right)\right|^{2} d r\right]^{\frac{1}{2}}, \quad 0<\theta<2 \pi,
$$

belongs to $L^{2}(0,2 \pi)$. The results of Riesz and $L i++l$ ewood and Paley were extended by many authors to harmonic functions and solutions of elliptic equations (for further historical material and bibliographic references see [10]). In particular Mikhailov in a sexies of the articles [7], [8], [9] and [10] extended the above results to solutions of the equation (1) under the assumption that $a_{i j} \in C^{I}(\bar{Q}), b_{i} \in C^{\perp}(\bar{Q})$ and $c \in C(\bar{Q})$. In this paper we establish Mikhailov's results under weaker assumptions, namely $b_{i} \in L^{s}(Q), s>n$, and $c \in L^{r}(Q), r>n / 2$, and by different methods. The plan of the paper is as follows. Section 1 is devoted to preliminaries. In Sections 2 and 3 we discuss traces of solutions in $W_{10 c}^{1,2}(Q)$ of (1). Section 4 deals with energy estimates for solutions in $W_{10 c}^{1,2}(Q)$. Finally in Section 5 we briefly study the Dirichlet problem in the $W_{l o c}^{1,2}$-framework. Recall that a weak solution in $W^{1,2}(Q)$ of the equation ( 1 ) is a solution of the Dirichlet problem with the boundary condition $u=\phi$ on $\partial Q$ if and only if $u-\phi_{1} \in \stackrel{\circ}{W}^{\mathcal{L}, 2}(Q)$. In this definition it is assumed that the boundary data $\phi$ is a trace of some function $\phi_{1}$ from $W^{1,2}(Q)$. Of course this assumption is rather restrictive, because not every function in $L^{2}(\partial Q)$ is the trace of some function belonging to $W^{1,2}(Q)$. In connection with the results obtained in Sections 2, 3 and 4 it makes sense to consider the Dirichlet problem with boundary data in $L^{2}(\partial Q)$. This possibility has already been noted by Nečas [12] and [13] (see Chapter 6) and by Mikhailov [9] and Guščin and 
Mikhailov [3].

\section{Preliminaries}

Let $Q \subset R_{n}$ be a bounded domain with the boundary $\partial Q$ of class $C^{2}$. In $Q$ we consider the equation ( 1 ).

We make the following assumptions:

(A) there exists a positive constant $\gamma$ such that

$$
\gamma^{-1}|\xi|^{2} \leq \sum_{i, j=1}^{n} a_{i j}(x) \xi_{i} \xi_{j} \leq \gamma|\xi|^{2}
$$

for all $x \in Q$ and $\xi \in R_{n} ;$ moreover the coefficients $a_{i j}$ are measurable and of class $c^{l}$ in some neighborhood of $\partial Q ;$

(B) $b_{i} \in L^{S}(Q) \quad(i=1, \ldots, n), c \in L^{r}(Q)$, where $n<s \leq \infty, n / 2<r \leq \infty ;$

(C) $\int_{Q} f(x)^{2} r(x)^{\theta} d x<\infty$, where $2 \leq \theta<3$, $r(x)=\operatorname{dist}(x, \partial Q)$.

In this paper we use the notion of a generalized solution of (1) involving the Sobolev spaces $W_{10 c}^{1,2}(Q), W^{l, 2}(Q)$ and $W^{l, 2}(Q)$ (for the definitions of these spaces see [2] or [4]).

A function $u(x)$ is said to be a weak solution of the equation (1) if $u \in W_{10 c}^{1,2}(Q)$ and $u$ satisfies

$$
\int_{Q}\left[\sum_{i, j=1}^{n} a_{i j}(x) D_{i} u D_{j} v+\sum_{i=1}^{n} b_{i}(x) D_{i} u \cdot v+c(x) u v\right] d x=\int_{Q} f(x) v d x
$$

for every $v \in W^{\perp, 2}(Q)$ with compact support in $Q$.

It follows from the regularity of the boundary $\partial Q$ that there is a number $\delta_{0}>0$ such that for $\delta \in\left(0, \delta_{0}\right]$ the domain $Q_{\delta}=Q \cap\left\{x ; \min _{y \in \partial Q}|x-y|>\delta\right\}$, with the boundary $\partial Q_{\delta}$, possesses the following 
property: to each $x_{0} \in \partial Q$ there is a unique point $x_{\delta}\left(x_{0}\right) \in \partial Q_{\delta}$ such that $x_{\delta}\left(x_{0}\right)=x_{0}-\delta \nu\left(x_{0}\right)$, where $\nu\left(x_{0}\right)$ is the outward normal to $\partial Q$ at $x_{0}$. The inverse mapping of $x_{0} \rightarrow x_{\delta}\left(x_{0}\right)$ is given by the formula $x_{0}=x_{\delta}+\delta v_{\delta}\left(x_{\delta}\right)$, where $v_{\delta}\left(x_{\delta}\right)$ is the outward normal to $\partial Q_{\delta}$ at $x_{\delta}$. Let $x_{\delta}$ denote an arbitrary point of $\partial Q_{\delta}$. For fixed $\delta \in\left(0, \delta_{0}\right]$ let

$$
\begin{aligned}
& A_{\varepsilon}=\partial Q_{\delta} \cap\left\{x ;\left|x-x_{\delta}\right|<\varepsilon\right\}, \\
& { }_{\varepsilon}=\left\{x ; x=\tilde{x}_{\delta}+\delta v_{\delta}\left(\tilde{x}_{\delta}\right), \tilde{x}_{\delta} \in A_{\varepsilon}\right\},
\end{aligned}
$$

and

$$
\frac{d S_{\delta}}{d S_{0}}=\lim _{\varepsilon \rightarrow 0} \frac{\left|A_{\varepsilon}\right|}{\left|B_{\varepsilon}\right|},
$$

where $|A|$ denote the $n-1$ dimensional Hausdorff measure of a set $A$. Mikhailov [8] proved that there is a positive number $\gamma_{0}$ such that

$$
\gamma_{0}^{-2} \leq \frac{d s_{\delta}}{d s_{0}} \leq \gamma_{0}^{2}
$$

and

$$
\lim _{\delta \rightarrow 0} \frac{d S_{\delta}}{d S_{0}}=1
$$

uniformly with respect to $x_{\delta} \in \partial Q_{\delta}$.

According to Lemma $I$ in [2], p. 382, the distance $r(x)$ belongs to $c^{2}\left(\bar{Q}-Q_{\delta_{0}}\right)$ if $\delta_{0}$ is sufficiently small. Denote by $\rho(x)$ the extension of the function $r(x)$ into $\bar{Q}$ satisfying the following properties $\rho(x)=r(x)$ for $x \in \bar{Q}-Q_{\delta_{0}}, \rho \in C^{2}(\bar{Q}), \quad \rho(x) \geq 3 \delta_{0} / 4$ in $Q_{\delta_{0}}$, $\gamma_{1}^{-1} r(x) \leq \rho(x) \leq \gamma_{1} r(x)$ in $Q$ for some positive constant $\gamma_{1}$, $\partial Q_{\delta}=\{x ; \rho(x)=\delta\}$ for $\delta \in\left\{0, \delta_{0}\right]$ and finally $\partial Q=\{x ; \rho(x)=0\}$. 
It follows from assumption (A) that the constant $\delta_{0}$ can be chosen so small that $a_{i j} \in C^{\mathcal{1}}\left(\overline{Q-Q_{\delta_{0}}}\right.$.

We will use the surface integrals

$$
M_{1}(\delta)=\int_{\partial Q}\left|u\left(x_{\delta}(x)\right)\right|^{2} d S_{x} \text { and } M(\delta)=\int_{\partial Q_{\delta}}|u(x)|^{2} d S_{x},
$$

where $u \in W_{l o c}^{I, 2}(Q)$ and the values of $u\left(x_{\delta}(x)\right)$ on $\partial Q$ and $u(x)$ on $\partial Q_{\delta}$ are understood in the sense of traces (see [4], Chapter 6). It follows from Lemma 4 in [1] that $M_{1}(\delta)$ and $M(\delta)$ are absolutely continuous on $\left[\delta_{1}, \delta_{0}\right]$ for every $0<\delta_{1}<\delta_{0}$. Moreover if $M(\delta)$ is bounded on $\left(0, \delta_{0}\right]$, then for every $0 \leq \alpha<1$ there is a positive constant $C$ such that

$$
\int_{Q_{\delta}} \frac{u(x)^{2}}{(\rho(x)-\delta)^{\alpha}} d x<C
$$

for every $\delta \in\left(0, \delta_{0} / 2\right]$ (for details see Lemma 5 in [1]).

The following result is a modification of Lemma 6 in [1].

LEMMA 1. Suppose that $u \in W_{10 \mathrm{C}}^{1,2}(Q)$ and that $\int_{Q}|D u(x)|^{2} r(x) d x<\infty$. Then if $0 \leq \mu<1$ and $0<\delta_{1} \leq \delta_{0} / 2$ we have, for $\delta \in\left(0, \delta_{1} / 2\right]$,

$$
\begin{aligned}
& \int_{Q_{\delta}} \frac{u(x)^{2}}{(\rho(x)-\delta)^{\mu}} d x \\
& \quad \leq K\left[\delta_{1}^{-\mu} \int_{Q_{\delta_{1}}} u(x)^{2} d x+\delta_{1}^{1-\mu} \int_{\partial Q_{\delta_{1}}} u(x)^{2} d S+\delta_{1}^{1-\mu} \int_{Q_{\delta}-Q_{\delta_{1}}}|D u(x)|^{2}(\rho(x)-\delta) d x\right],
\end{aligned}
$$

where $K$ is a positive constant independent of $\delta_{1}$ and $\delta$.

Proof. Let $\delta \in\left(0, \delta_{1} / 2\right]$ and put

$$
\int_{Q_{\delta}} \frac{u^{2}}{(\rho-\delta)^{\mu}} d x=\int_{Q_{\delta}-Q_{\delta_{1}}} \frac{u^{2}}{(\rho-\delta)^{\mu}} d x+\int_{Q_{\delta_{1}}} \frac{u^{2}}{(\rho-\delta)^{\mu}} d x
$$


Since $\rho(x) \geq \delta_{1}$ on $Q_{\delta_{1}}$, we have

$$
\int_{Q_{\delta_{1}}} \frac{u^{2}}{(\rho-\delta)^{\mu}} d x \leq\left(2 / \delta_{1}\right)^{\mu} \int_{Q_{\delta_{1}}} u^{2} d x
$$

We now note that

$$
\begin{aligned}
\int_{Q_{\delta}-Q_{\delta}} & \frac{u^{2}}{(\rho-\delta)^{\mu}} d x=\int_{\delta}^{\delta}(t-\delta)^{-\mu} \int_{\partial Q} u\left(x_{t}\left(x_{0}\right)\right)^{2} \frac{d S_{t}}{d S_{0}} d S_{0} d t \\
& \leq \gamma_{0}^{2} \int_{\delta}^{\delta}(t-\delta)^{-\mu} \int_{\partial Q} u\left(x_{t}\left(x_{0}\right)\right)^{2} d S_{0} d t .
\end{aligned}
$$

As $\int_{\partial Q} u\left(x_{t}(x)\right)^{2} d s_{x}$ is absolutely continuous on $\left[\delta, \delta_{1}\right]$, integrating by parts

$$
\begin{aligned}
& \int_{Q_{\delta}-Q_{\delta_{1}}} \frac{u^{2}}{(\rho-\delta)^{\mu}} d x \\
& \leq \frac{\gamma_{0}^{2} \delta_{1}^{1-\mu}}{1-\mu} \int_{\partial Q} u\left(x_{\delta_{1}}(x)\right)^{2} d S \\
& +\frac{2 \gamma_{0}^{2}}{1-\mu} \int_{\delta}^{\delta_{1}}(t-\delta)^{1-\mu} \int_{\partial Q}\left|u\left(x_{t}\left(x_{0}\right)\right)\right|\left|D u\left(x_{t}\left(x_{0}\right)\right)\right|\left|\frac{\partial}{\partial t} x_{t}\left(x_{0}\right)\right| d s_{0} d t \\
& \leq \frac{\gamma_{0}^{4} \delta_{1}^{1-\mu}}{1-\mu} \int_{\partial Q_{\delta_{1}}} u^{2} d s+\frac{2 \gamma_{0}^{4}}{1-\mu} \int_{Q_{\delta}-Q_{\delta_{1}}}|u(x)||D u(x)|\left(\rho_{-} \delta\right)^{1-\mu} d x \\
& \leq \frac{\gamma_{0}^{4} \delta_{1}^{1-\mu}}{1-\mu} \int_{\partial Q_{\delta_{1}}} u^{2} d S+\frac{2 \beta \gamma_{0}^{4}}{1-\mu} \int_{Q_{\delta}-Q_{\delta_{1}}} \frac{u^{2}}{(\rho-\delta)^{\mu}} d x \\
& +\frac{2 \gamma_{0}^{4} \delta_{1}^{1-\mu}}{\beta(1-\mu)} \int_{Q_{\delta}-Q_{\delta}}|D u|^{2}(\rho-\delta) d x
\end{aligned}
$$

where we have used Young's inequality in the final step. Now choosing 
$2 \gamma_{0}^{4 \beta} /(1-\mu)=\frac{1}{2}$ the result follows.

\section{Main result}

We are now in a position to establish a criterion for the continuity of $M_{1}(\delta)$ and $M(\delta)$ on $\left[0, \delta_{0}\right]$ which plays an essential part in the ensuing treatment of the Dirichlet problem.

THEOREM 1. Let $u$ be a solution of (1) belonging to $W_{10 \mathrm{c}}^{1,2}(Q)$, then the following conditions are equivalent:

I. $M(\delta)$ is a bounded function on $\left(0, \delta_{0}\right]$;

II. $\int_{Q}|D u(x)|^{2} r(x) d x<\infty$;

III. $M_{1}(\delta)$ is continuous on $\left[0, \delta_{0}\right]$.

Proof. Let $\Phi$ be a smooth function on $\bar{Q}$ such that $\Phi \equiv 1$ on $Q-Q_{\delta_{1} / 2}, \Phi=0$ on $Q_{\delta_{1}}$ and $0 \leq-\Phi \leq 1$ on $\bar{Q}$, where $0<\delta_{1} \leq \delta_{0} / 2$ is to be determined later. Put

$$
v(x)= \begin{cases}u(x)(\rho(x)-\delta) \Phi(x)^{2} & \text { for } x \in Q_{\delta}, \\ 0 & \text { for } x \in Q-Q_{\delta},\end{cases}
$$

where $0<\delta \leq \delta_{0} / 2$. It is clear that $v$ is an admissible test function in (2) and

(6) $\int_{Q_{\delta}} \sum_{i, j=1}^{n} a_{i j} D_{i} u D_{j} u(\rho-\delta) \Phi^{2} d x+\int_{Q_{\delta}} \sum_{i, j=1}^{n} a_{i j} D_{i} u \cdot u D_{j} \rho \Phi^{2} d x$

$$
\begin{aligned}
& +2 \int_{Q_{\delta}} \sum_{i, j=1}^{n} a_{i j} D_{i} u \cdot u(\rho-\delta) \Phi D_{j} \Phi d x+\int_{Q_{\delta}} \sum_{i=1}^{n} b_{i} D_{i} u \cdot u(\rho-\delta) \Phi^{2} d x \\
& +\int_{Q_{\delta}} c u^{2}(\rho-\delta) \Phi^{2} d x \\
& =\int_{Q_{\delta}} f u(\rho-\delta) \Phi^{2} d x .
\end{aligned}
$$


The proof of $I \Rightarrow I I$. Denote the integrals on the left hand side of (6) by $J_{1}, J_{2}, J_{3}, J_{4}$ and $J_{5}$. It follows from assumption (A) that

$$
J_{1} \geq \gamma^{-1} \int_{Q_{\delta}}|D u|^{2}(\rho-\delta) \Phi^{2} d x
$$

By Green's formula (see [11], p. 139) we have

$$
\begin{aligned}
J_{2} & =\frac{1}{2} \int_{Q_{\delta}} \sum_{i, j=1}^{n} a_{i j} D_{i} u^{2} D_{j} \rho \Phi^{2} d x \\
& =-\frac{1}{2} \int_{\partial Q_{\delta}} \sum_{i, j=1}^{n} a_{i j j} D_{i} \rho D_{j} \rho u^{2} \Phi^{2} d S-\frac{1}{2} \int_{Q_{\delta}} \sum_{i, j=1}^{n} D_{i}\left(a_{i j} D_{j} \rho \Phi^{2}\right) u^{2} d x .
\end{aligned}
$$

Thus

$$
\text { (8) } \begin{aligned}
\left|J_{2}\right| \leq \frac{1}{2} \int_{\partial Q_{\delta}}\left(\sum_{i, j=1}^{n} a_{i j} D_{i} \rho D_{j} \rho\right) u^{2} \Phi^{2} d S+C_{1} \int_{Q_{\delta}} u^{2} \Phi^{2} d x & +C_{2} \int_{Q_{\delta}} u^{2}\left|D_{x} \Phi\right| \Phi d x,
\end{aligned}
$$

where

$$
C_{1}=\frac{1}{2} \sup _{Q-Q_{\delta_{0}}} \sum_{i, j=1}^{n}\left|D_{i}\left(a_{i j} D_{j} \rho\right)\right|, C_{2}=\sup _{Q} \sum_{i, j=1}^{n}\left|a_{i j} D_{j} \rho\right| .
$$

It follows from Young's inequality that

$$
\left|J_{3}\right| \leq \frac{\Upsilon^{-1}}{8} \int_{Q_{\delta}}|D u|^{2}(\rho-\delta) \Phi^{2} d x+C_{3} \int_{Q_{\delta}} u^{2}(\rho-\delta)\left|D_{x} \Phi\right|^{2} d x,
$$

where

$$
c_{3}=8 r \sup _{Q} \sum_{i, j=1}^{n}\left|a_{i j}\right| \text {. }
$$

To estimate $J_{4}$ and $J_{5}$ let us first suppose that $n>3$. In view of Hölder's inequality we obtain 
Boundary values of elliptic equations

9

$$
\begin{aligned}
\left|J_{4}\right| \leq\left\|\sum_{i=1}^{n} b_{i}\right\|_{L^{s}(Q)}\left\|(\rho-\delta)^{-\varepsilon}\right\|_{L^{1}{ }^{1}\left(Q_{\delta}-Q_{\delta}\right)} & \\
& \times\left\|u(\rho-\delta)^{\frac{1}{2}+\varepsilon} \Phi\right\|_{L^{2 *}}\left(Q_{\delta}\right) \\
& \left\|D_{x} u(\rho-\delta)^{\frac{1}{2}} \Phi\right\|_{L^{2}\left(Q_{\delta}\right)},
\end{aligned}
$$

where

$$
\frac{1}{2^{*}}=\frac{1}{2}-\frac{1}{n}, \frac{1}{s}+\frac{1}{s_{1}}=\frac{1}{n},
$$

and $0<\varepsilon<1$ is chosen so that $\varepsilon s_{1}<1$. In view of $(5)$,

$$
\int_{Q_{\delta}} \frac{|u(x)|^{2}}{(\rho(x)-\delta)^{\alpha}} d x \leq c, 0<\alpha<1 \text {, }
$$

for $\delta \in\left(0, \delta_{0} / 2\right)$ and consequently $u(\rho-\delta)^{\frac{1}{2}+\varepsilon} \in \mathcal{O}^{l, 2}\left(Q_{\delta}\right)$. By Sobolev's inequality ([2], p. 148) we have

$$
\begin{aligned}
& \left\|u(\rho-\delta)^{\frac{1}{2}+\varepsilon} \Phi\right\|_{L^{2 *}}\left(Q_{\delta}\right) \\
& \leq S\left[\left\|D_{x} u(\rho-\delta)^{\frac{1}{2}+\varepsilon_{\Phi}}\right\|_{L^{2}\left(Q_{\delta}\right)}+\left(\frac{1}{2}+\varepsilon\right)\left\|u \mid D_{x}^{\rho \mid(\rho-\delta)^{\varepsilon-\frac{1}{2}} \Phi}\right\|_{L^{2}\left(Q_{\delta}\right)}+\left\|u(\rho-\delta)^{\frac{1}{2}+\varepsilon_{D}} x^{\Phi}\right\|_{L^{2}\left(Q_{\delta}\right)}\right]
\end{aligned}
$$

for some positive constant $S$ independent of $\delta$. Therefore there is a positive constant $\tilde{S}$ such that

$$
\begin{aligned}
& \left|J_{4}\right| \leq \tilde{S}\left\|\sum_{i=1}^{n} b_{i}\right\|_{L^{s}(Q)}\left\|(\rho-\delta)^{-\varepsilon}\right\|_{L^{s}{ }^{1}\left(Q_{\delta}-Q_{\delta_{1}}\right)} \\
& \times\left[\int_{Q_{\delta}}\left|D x^{u}\right|^{2}(\rho-\delta) \Phi^{2} d x+\left\|D_{x} u(\rho-\delta)^{\frac{1}{2}} \Phi\right\|_{L^{2}\left(Q_{\delta}\right)}\left\|u(\rho-\delta)^{\varepsilon-\frac{1}{2}} \Phi\right\|_{L}^{2}\left(Q_{\delta}\right)\right. \\
& \left.+\left\|D_{x} u(\rho-\delta)^{\frac{1}{2}} \Phi\right\|_{L^{2}\left(Q_{\delta}\right)}\left\|u(\rho-\delta)^{\frac{1}{2}} D_{x} \Phi\right\|_{L^{2}\left(Q_{\delta}\right)}\right] \text {. }
\end{aligned}
$$

Note that there is a constant $A>0$ independent of $\delta$ such that

https://doi.org/10.1017/S0004972700011461 Published online by Cambridge University Press 
10

J. Chabrowski and H.B. Thompson

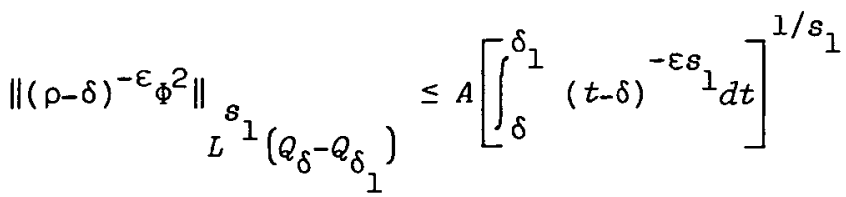

$$
\begin{aligned}
& =A\left(1-\varepsilon s_{1}\right)^{-1 / s_{1}} \delta_{1}\left(1-\varepsilon s_{1}\right) / s_{1} .
\end{aligned}
$$

Choose $\delta_{1}$ so that

$$
A \tilde{S}\left(1-\varepsilon s_{1}\right)^{-1 / s_{1}} \delta_{1}\left(1-\varepsilon s_{1}\right) / s\left\|_{1} \sum_{i=1}^{n} b_{i}\right\|_{L(Q)} \leq \frac{\gamma^{-1}}{8} .
$$

Consequently by Young's inequality we obtain

(10) $\left|J_{4}\right| \leq \frac{3 \gamma^{-1}}{8} \int_{Q_{\delta}}\left|D_{x} u\right|^{2}(\rho-\delta) \Phi^{2} d x+\frac{\gamma^{-1}}{8} \int_{Q_{\delta}} u^{2}(\rho-\delta)^{2 \varepsilon-1} d x$

$$
+\frac{\gamma^{-1}}{8} \int_{Q_{\delta}} u^{2}(0-\delta)\left|D_{x} \Phi\right|^{2} d x
$$

Similarly

$$
\left|J_{5}\right| \leq\|c\|_{L^{r}(Q)}\left\|(\rho-\delta)^{-2 \varepsilon_{1}}\right\|_{L^{r}{ }^{r}\left(Q_{\delta}-Q_{\delta_{1}}\right)}\left\|u(\rho-\delta)^{\frac{1}{2}+\varepsilon_{1}}{ }\right\|_{L^{2 *}\left(Q_{\delta}\right)}^{2},
$$

where

$$
\frac{1}{r}+\frac{1}{r_{1}}=\frac{2}{n}, \quad \varepsilon_{1} r_{1}<1 \text {. }
$$

Then in view of the Sobolev inequality there is a positive constant $S_{1}$ such that

$$
\begin{aligned}
\left|J_{5}\right| \leq & S_{1}\|c\|_{L}{ }^{r}(Q) \\
& \times\left[\int_{Q_{\delta}} u^{2}(\rho-\delta)^{-1+2 \varepsilon}{ }^{-2 \varepsilon} d x+\int_{Q^{1}} \mid(\rho) Q_{\delta}-Q_{\delta_{1}}\right) \\
& \left.|D u|^{2}(\rho-\delta) \Phi^{2} d x+\int_{Q_{\delta}} u^{2}(\rho-\delta)\left|D_{x} \Phi\right|^{2} d x\right]
\end{aligned}
$$

Finally taking $\delta_{1}$ so small that

https://doi.org/10.1017/S0004972700011461 Published online by Cambridge University Press 


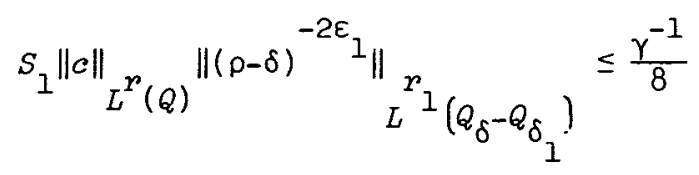

we arrive at the inequality

$$
\begin{aligned}
\left|J_{5}\right| \leq \frac{\gamma^{-1}}{8} \int_{Q_{\delta}}\left|D_{x} u\right|^{2}(\rho-\delta) \Phi^{2} d x+\frac{\gamma^{-1}}{8} \int_{Q_{\delta}} u^{2}(\rho-\delta)^{-1+2 \varepsilon} 1 d x \\
\quad+\frac{\gamma^{-1}}{8} \int_{Q_{\delta}} u^{2}(\rho-\delta)\left|D_{x} \Phi\right|^{2} d x .
\end{aligned}
$$

The remaining case $n=2$ can now be obtained by using the fact that $W^{l, 2}(Q) \quad(n=2)$ is continuously imbedded in $L^{q}(Q)$ for all $I \leq q<\infty$ (see $[4]$, p. 287).

By Young's inequality we obtain

$$
\left|\int_{Q_{\delta}} f u(\rho-\delta) \Phi^{2} d x\right| \leq \frac{1}{2} \int_{Q_{\delta}} f^{2}(\rho-\delta)^{\theta} d x+\frac{1}{2} \int_{Q_{\delta}} u^{2}(\rho-\delta)^{-\alpha} d x,
$$

where $\alpha=\theta-1<1$. Combining the identity (6) and the estimates (7), $(8),(9),(10),(11)$ and (12) we deduce that there is a positive constant $C_{4}$ such that

$$
\begin{aligned}
\int_{Q_{\delta}}|D u|^{2}(\rho-\delta) \Phi^{2} d x \leq C_{4}\left[M(\delta)+\int_{Q_{\delta}} u^{2} d x\right. & +\int_{Q_{\delta}} u^{2}(\rho-\delta)\left|D_{x} \Phi\right|^{2} d x \\
& \left.+\int_{Q_{\delta}} u^{2}(\rho-\delta)^{-\beta} d x+\int_{Q_{\delta}} f^{2}(\rho-\delta)^{\theta} d x\right],
\end{aligned}
$$

for all $\delta \in\left(0, \delta_{1} / 2\right]$, where $\beta=\max \left(\alpha, 1-2 \varepsilon_{1}, 1-2 \varepsilon\right)$. The Monotone Convergence Theorem implies that

$$
\int_{Q-Q_{\delta_{1} / 2}}|\operatorname{Du}(x)|^{2} r(x) d x<\infty .
$$

Since $u \in W_{l o c}^{1,2}(Q)$, it follows that $I \Rightarrow I I$.

To prove II $\Rightarrow$ III note that Lemma 1 implies 


$$
\int_{Q_{\delta}} \frac{u(x)^{2}}{(\rho(x)-\delta)^{\mu}} d x \leq c, 0 \leq \mu<1,
$$

for $\delta \in\left(0, \delta_{0} / 2\right]$, where $C$ is independent of $\delta$. First we prove that $M(\delta)$ is continuous at $\delta=0$. Indeed from the first part of the proof

$$
\begin{aligned}
& \frac{1}{2} \int_{\partial Q_{\delta}} \sum_{i, j=1}^{n} a_{i j} D_{i} \rho D D_{j} \rho u^{2} \Phi^{2} d S=\int_{Q_{\delta}} \sum_{i, j=1}^{n} a_{i j} D_{i} u D j u(\rho-\delta) \Phi^{2} d x \\
&-\frac{1}{2} \int_{Q_{\delta}} \sum_{i, j=1}^{n} D_{i}\left(a_{i j} D_{j} \rho \Phi^{2}\right) u^{2} d x+2 \int_{Q_{\delta}} \sum_{i, j=1}^{n} a_{i j} D_{i} u u(\rho-\delta) \Phi D, \Phi d x \\
&+\int_{Q_{\delta}} \sum_{i=1}^{n} b_{i} D_{i} u u(\rho-\delta) \Phi^{2} d x+\int_{Q_{\delta}} c u^{2}(\rho-\delta) \Phi^{2} d x-\int_{Q_{\delta}} f u(\rho-\delta) \Phi^{2} d x .
\end{aligned}
$$

Thus

$$
\lim _{\delta \rightarrow 0} \int_{\partial Q_{\delta}} u^{2} \sum_{i, j=1}^{n} a_{i j} D_{i} \rho D_{j} \rho d S_{x}
$$

exists, by the Dominated Convergence Theorem. Since

$$
\gamma^{-1} \leq \sum_{i, j=1}^{n} a_{i j}{ }_{i} \rho D_{i} \rho \leq \gamma
$$

is continuous on $\overline{Q-Q}_{\delta_{0}}$ it follows that $M(\delta)$ is continuous at $\delta=0$.

That II $\Rightarrow$ III follows from the relationship

$$
M(\delta)-M_{1}(\delta)=\int_{\partial Q} u\left(x_{\delta}(x)\right)^{2}\left[\frac{d S_{\delta}}{d S_{0}}-1\right] d S,
$$

since $d S_{\delta} / d S_{0} \rightarrow 1$ uniformly as $\delta \rightarrow 0$.

Finally III $\Rightarrow$ I follows from the proof II $\Rightarrow$ III.

3. Traces in $L^{2}(\partial Q)$

Our next objective is to prove that $u$ has a trace on $\partial Q$ in $L^{2}(\partial Q)$; that is, $u\left(x_{\delta}\right)$ converges in $L^{2}(\partial Q)$ as $\partial \rightarrow 0$. To do this we 
first show that $u\left(x_{\delta}\right)$ converges weakly in $L^{2}(\partial Q)$ to some function $\zeta$ (Theorems 2 and 3) and then show norm $u\left(x_{\delta}\right)$ converges to norm $\zeta$. The result then follows by uniform convexity.

THEOREM 2. Let $u \in W_{10 c}^{1,2}(Q)$ be a solution of (1). Assume that one of the conditions I, II or III holds. Then there is a sequence $\delta_{\nu} \rightarrow 0$ as $\nu \rightarrow \infty$ and a function $\zeta \in L^{2}(\partial Q)$ such that

$$
\lim _{\nu \rightarrow \infty} \int_{\partial Q} u\left(x_{\delta_{\nu}}(x)\right) g(x) d S_{x}=\int_{\partial Q} \zeta(x) g(x) d S_{x}
$$

for each $g \in L^{2}(\partial Q)$.

THEOREM 3. Let $u \in W_{10 c}^{1,2}(Q)$ be a solution of (1). If one of the conditions I, II or III holds, then the function

$$
G(\delta)=\int_{\partial Q} u\left(x_{\delta}(x)\right) \Psi(x) d S_{x}
$$

is continuous on $\left[0, \delta_{0}\right]$ for every $\Psi$ in $L^{2}(\partial Q)$.

Proof. It is clear that $G(\delta)$ is absolutely continuous on $\left[\delta_{1}, \delta_{0}\right]$ for any $\delta_{1}<\delta_{0}$, hence it remains to prove the continuity at $\delta=0$. Note that

$$
g(x)=\sum_{i, j=1}^{n} a_{i j}(x) D_{i} \rho D_{j} \rho
$$

is uniformly continuous on $\overline{Q-Q}_{\delta_{0}}$ and $\gamma^{-1} \leq g(x) \leq \gamma$ on $\bar{Q}$. On the other hand $M_{1}(\delta)$ is bounded and the elements of $C^{\mathcal{l}}(\bar{Q})$ vanishing on $Q_{\delta_{0}}$ are dense in $L^{2}(\partial Q)$, so it suffices to show that

$$
\bar{G}(\delta)=\int_{\partial Q_{\delta}} u \Psi \sum_{i, j=1}^{n} a_{i j} D_{i} \rho D_{j} \rho d S_{x}
$$

is continuous for each $\Psi \in C^{l}(\bar{Q})$ vanishing on $Q_{\delta_{0}}$. 
Taking

$$
v= \begin{cases}\Psi(\rho-\delta) & \text { for } x \in Q_{\delta}, \\ 0 & \text { for } x \in Q-Q_{\delta},\end{cases}
$$

in (2) as a test function, we obtain

$$
\begin{aligned}
\bar{G}(\delta)=\int_{Q_{\delta}}\left[\sum_{i, j=1}^{n} a_{i j} D_{i} u D_{j} \Psi(\rho-\delta)\right. & -\sum_{i, j=1}^{n} D_{i}\left(a_{i j} D_{j} \rho \Psi\right) u \\
& \left.+\sum_{i=1}^{n} b_{i} D_{i} u \Psi(\rho-\delta)+c u \Psi(\rho-\delta)-f \Psi(\rho-\delta)\right] d x .
\end{aligned}
$$

By an argument similar to that used in the proof of Theorem 1 one can easily show that the integrand on the right hand side is in $L^{1}(Q)$ and the result follows.

In order to prove the convergence of the norm we use the following function and technical lemmas.

For $\delta \in\left(0, \delta_{0}\right]$ we define the mapping $x^{\delta}: \bar{Q} \rightarrow \bar{Q}_{\delta}$ by

$$
x^{\delta}(x)= \begin{cases}x & \text { for } x \in Q_{\delta}, \\ x_{\delta}+\frac{1}{2}\left(x-x_{\delta}\right) & \text { for } x \in Q-Q_{\delta} .\end{cases}
$$

Thus $x^{\delta}(x)=x$ for each $x \in Q_{\delta}$ and $x^{\delta}(x)=x_{\delta / 2}(x)$ for each $x \in \partial Q$. Moreover $\rho\left(x^{\delta}\right) \geq \delta / 2$ and $x^{\delta}$ is uniformly Lipschitz continuous. Note that if $u \in W_{100}^{1,2}(Q)$ then $u\left(x^{\delta}\right) \in W^{1,2}(Q)$.

The proofs of the following lemmas can be found in [1].

LEMMA 2. Let $h \in L^{1}(Q)$, then

$$
\lim _{\delta \rightarrow 0} \int_{Q-Q_{\delta}} h\left(x^{\delta}(x)\right) d x=0
$$

LEMMA 3. If $\rho^{\mu / 2} f \in L^{2}(Q), 0 \leq \mu<1, g \in L^{2}(Q)$ and $\int_{\partial Q_{\delta}} g(x)^{2} d S_{x}$ is bounded on $\left(0, \delta_{0}\right]$, then 
Boundary values of elliptic equations

15

$$
\lim _{\delta \rightarrow 0} \int_{Q-Q_{\delta}} g\left(x^{\delta}(x)\right) f(x) d x=0
$$

LEMMA 4. If $f$ is a nonnegative function in $L^{1}(Q)$ and if $\int_{\partial Q_{\delta}} f(x) d S_{x}$ is bounded on $\left(0, \delta_{0}\right]$, then

$$
\int_{Q-Q_{\delta}} \frac{f\left(x^{\delta}(x)\right)}{\rho(x)^{\mu}} d x \leq \frac{\gamma_{0}^{4} \delta^{1-\mu}}{1-\mu} \sup _{\left(0, \delta_{0}\right]} \int_{\partial Q_{t}} f(x) d S_{x},
$$

where $0 \leq \mu<1$.

LEMMA 5. Let $g \in L^{2}(Q), \rho^{\frac{1}{2}} f \in L^{2}(Q)$ and suppose that $\int_{\partial Q_{\delta}}|g(x)|^{2} d S_{x}$ is bounded on $\left[0, \delta_{0}\right]$, then

$$
\lim _{\delta \rightarrow 0} \int_{Q-Q_{\delta}} f\left(x^{\delta}(x)\right) g(x) d x=0
$$

LEMMA 6. If $\rho^{\frac{1}{2}} f$ and $\rho^{\frac{1}{2}} g$ belong to $L^{2}(Q)$, then

$$
\lim _{\delta \rightarrow 0} \int_{Q-Q_{\delta}} f\left(x^{\delta}(x)\right) g(x) p(x) d x=0 \text {. }
$$

Let $L_{1}^{2}=L^{2}\left(\partial Q, d S_{x}\right)$ with inner product (norm) denoted by $\langle\cdot, \cdot\rangle_{1}$ $\left(\|\cdot\|_{1}\right)$ and $L_{2}^{2}=L^{2}\left(\partial Q, g d S_{x}\right)$ with inner product (norm) denoted by $(\cdot, \cdot)_{2}\left(\|\cdot\|_{2}\right)$, where

$$
g(x)=\sum_{i, j=1}^{n} a_{i j}(x) D_{i} \rho(x) D_{j} \rho(x) .
$$

Now we are in a position to prove the main result of this section.

THEOREM 4. Let $u \in W_{1 \mathrm{loc}}^{1,2}(Q)$ be a solution of (I) such that one of the conditions I, II or III holds. Then there is a function $\zeta$ belonging to $L^{2}(\partial Q)$ such that $u\left(x_{\delta}\right)$ converges to $\zeta$ in $L_{1}^{2}$.

https://doi.org/10.1017/S0004972700011461 Published online by Cambridge University Press 
Proof. Since $\|\cdot\|_{1}$ and $\|\cdot\|_{2}$ are equivalent it suffices to show that there is $\zeta \in L_{2}^{2}$ such that $\lim _{\delta \rightarrow 0} u\left(x_{\delta}\right)=\zeta$ in $L_{2}^{2}$. By Theorem 2 and 3 there is $\zeta \in L_{2}^{2}$ such that $\lim _{\delta \rightarrow 0} u\left(x_{\delta}\right)=\zeta$ weakly in $L_{2}^{2}$. Since $L_{2}^{2}$ is uniformly convex it suffices to show that $\underset{\delta \rightarrow 0}{\lim }\left\|u\left(x_{\delta}\right)\right\|_{2}=\|\zeta\|_{2} \cdot$ Let $\Psi \in W^{1,2}(Q)$ and $\Psi \equiv 0$ on $Q_{\delta_{0}}$, set $F(\Psi(x))=\sum_{i, j=1}^{n} a_{i j} D_{i} u D_{j} \Psi \rho-\sum_{i, j=1}^{n} D_{i}\left(a_{i j} D_{j} \rho \Psi\right) u$ $+\sum_{i=1}^{n} b_{i} D_{i} u \Psi \rho+c u \Psi \rho-f u \Psi \rho$

As in the proof of Theorem 1 we find that

$$
\langle\zeta, \Psi\rangle_{2}=\int_{Q} F(\Psi(x)) d x
$$

for all $\Psi \in C^{\perp}(\bar{Q})$ such that $\Psi \equiv 0$ on $Q_{\delta_{0}}$ and hence for all $\Psi \in W^{l, 2}(Q)$ with $\Psi \equiv 0$ on $Q_{\delta_{0}}$. Let $\Phi$ be as defined in the proof of Theorem 1. Since $u\left(x^{\delta}\right) \Phi(x)^{2} \in W^{1,2}(Q)$ and $u\left(x^{\delta}\right) \Phi(x)^{2}=0$ on $Q_{0}$ we have

$\left\langle\zeta, u\left(x^{\delta}\right)\right\rangle_{2}=\int_{Q} F\left(u\left(x^{\delta}(x)\right)\right) d x=\int_{Q-Q_{\delta}} F\left(u\left(x^{\delta}(x)\right)\right) d x+\int_{Q_{\delta}} F\left(u(x) \Phi(x)^{2}\right) d x$, for $\delta \leq \delta_{1} / 2$, since $x^{\delta}(x)=x$ on $Q_{\delta}$ and $\Phi \equiv 1$ on $Q-Q_{\delta_{1} / 2}$. We show that

$$
\lim _{\delta \rightarrow 0} \int_{Q-Q_{\delta}} F\left(u\left(x^{\delta}(x)\right)\right) d x=0
$$

and that 


$$
\lim _{\delta \rightarrow 0} \int_{Q_{\delta}} F\left(u(x) \Phi(x)^{2}\right) d x=\lim _{\delta \rightarrow 0}\left\|u\left(x_{\delta}\right)\right\|_{2}^{2}
$$

so that

$$
\|\zeta\|_{2}^{2}=\lim _{\delta \rightarrow 0}\left(\zeta, u\left(x^{\delta}\right)\right\rangle=\lim _{\delta \rightarrow 0}\left\|u\left(x^{\delta}\right)\right\|_{2}^{2}
$$

since $x^{\delta}(x)=x_{\delta / 2}(x)$ on $\partial Q$. Setting

$$
v(x)= \begin{cases}u(x)(\rho(x)-\delta) \Phi(x)^{2} & \text { for } x \in Q_{\delta}, \\ 0 & \text { for } x \in Q-Q_{\delta},\end{cases}
$$

it follows from (6) that

$$
\begin{aligned}
& \lim _{\delta \rightarrow 0} \int_{Q_{\delta}} F\left(u \Phi^{2}\right) d x=\lim _{\delta \rightarrow 0} \int_{Q_{\delta}}\left[\sum_{i, j=1}^{n} a_{i j} D_{i} u D_{j} u(\rho-\delta) \Phi^{2}\right. \\
& +2 \sum_{i, j=1}^{n} a_{i j} D_{i} u u \Phi D_{j} \Phi(\rho-\delta)-\sum_{i, j=1}^{n} D_{i}\left(a_{i j} D_{j} \rho \Phi^{2} u\right) u \\
& \left.+\sum_{i=1}^{n} b_{i} D_{i} u u(\rho-\delta) \Phi^{2}+c u^{2}(\rho-\delta) \Phi^{2}-f u(\rho-\delta) \Phi^{2}\right] d x \\
& =-\lim _{\delta \rightarrow 0} \int_{Q_{\delta}} \sum_{i, j=1}^{n} D_{i}\left(a_{i j} D_{j} \rho \Phi^{2} u^{2}\right) d x \\
& =\lim _{\delta \rightarrow 0} \int_{\partial Q_{\delta}} u^{2} g d S_{x} \\
& =\lim _{\delta \rightarrow 0} \int_{\partial Q} u\left(x_{\delta}\right)^{2} g(x) d S_{x}+\lim _{\delta \rightarrow 0} \int_{\partial Q} u\left(x_{\delta}\right)^{2}\left[g\left(x_{\delta}\right)-g(x)\right] d S_{x} \\
& =\lim _{\delta \rightarrow 0} \int_{\partial Q} u\left(x_{\delta}\right)^{2} g(x) d S_{x} \\
& =\lim _{\delta \rightarrow 0}\left\|u\left(x_{\delta}\right)\right\|_{2}^{2} \text {. }
\end{aligned}
$$

Now it remains to prove that $\lim _{\delta \rightarrow 0} \int_{Q-Q_{\delta}} F\left(u\left(x^{\delta}(x)\right)\right) d x=0$. Note that 
18

J. Chabrowski and H.B. Thompson

$$
\begin{aligned}
& \left|F\left(u\left(x^{\delta}(x)\right)\right)\right| \\
& \leq C\left[|D u(x)|\left|D u\left(x^{\delta}(x)\right)\right| \rho(x)+|u(x)|\left|u\left(x^{\delta}(x)\right)\right|+\left|D u\left(x^{\delta}(x)\right)\right||u(x)|\right] \\
& +\sum_{i=1}^{n}\left|b_{i}(x)\right||D u(x)|\left|u\left(x^{\delta}(x)\right)\right| \rho(x)+|c(x)||u(x)|\left|u\left(x^{\delta}(x)\right)\right| \rho(x) \\
& +|f(x)|\left|u\left(x^{\delta}(x)\right)\right| \rho(x),
\end{aligned}
$$

for $x \in Q-Q_{\delta}$, where $C$ is a positive constant independent of $\delta$. Since $\rho^{(\theta / 2)-1}(\rho f) \in L^{2}, \quad 0 \leq \theta / 2-1<\frac{1}{2}$ and $\int_{\partial Q_{\delta}} u^{2} d S_{x}$ is bounded, by Lemma 3 ,

$$
\lim _{\delta \rightarrow 0} \int_{Q-Q_{\delta}}|f(x)|\left|u\left(x^{\delta}\right)\right| \rho(x) d x=0
$$

It follows from Lemma 2 and Hölder's inequality that

$$
\lim _{\delta \rightarrow 0} \int_{Q-Q_{\delta}}\left|u(x) \| u\left(x^{\delta}\right)\right| \rho(x) d x=0 \text {. }
$$

In view of Lemmas 5 and 6 ,

$$
\begin{aligned}
\lim _{\delta \rightarrow 0} \int_{Q-Q_{\delta}}\left|D u\left(x^{\delta}(x)\right) \| u(x)\right| d x & =0, \\
\lim _{\delta \rightarrow 0} \int_{Q-Q_{\delta}}|D u(x)|\left|D u\left(x^{\delta}(x)\right)\right| \rho(x) d x & =0 .
\end{aligned}
$$

To estimate the remaining terms we restrict ourselves to the case $n>2$. By Hölder's inequality

$$
\begin{aligned}
& \int_{Q-Q_{\delta}} \sum_{i=1}^{n}\left|b _ { i } ( x ) \left\||D u(x) \|| u\left(x^{\delta}(x)\right) \mid \rho(x) d x\right.\right.
\end{aligned}
$$

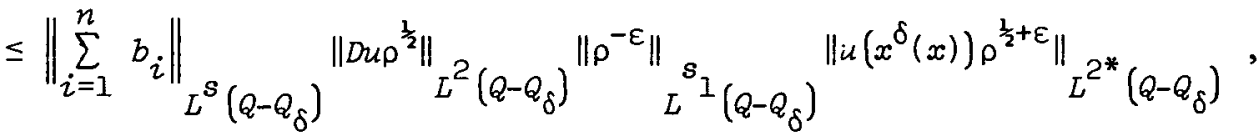

where

$$
\frac{1}{s}+\frac{1}{s_{1}}=\frac{1}{n}, \frac{1}{2^{*}}=\frac{1}{2}-\frac{1}{n}, \varepsilon s_{1}<1 .
$$

https://doi.org/10.1017/S0004972700011461 Published online by Cambridge University Press 
Now the Sobolev inequality implies that

$$
\left\|u\left(x^{\delta}(x)\right) \rho^{\frac{1}{2}+\varepsilon}\right\|_{L^{2^{*}}} \leq S\left[\left\|D u\left(x^{\delta}(x)\right) \rho^{\frac{1}{2}+\varepsilon}\right\|_{L^{2}\left(Q-Q_{\delta}\right)}+\left\|u\left(x^{\delta}(x)\right) \rho^{-\frac{1}{2}+\varepsilon}\right\|_{L^{2}\left(Q-Q_{\delta}\right.}\right]
$$

and consequently, by Lemmas 4 and 6 ,

$$
\lim _{\delta \rightarrow 0} \int_{Q-Q_{\delta}} \sum_{i=1}^{n}\left|b_{i}(x)\right||D u(x)|\left|u\left(x^{\delta}(x)\right)\right| \rho(x) d x
$$

Similarly one can prove that

$$
\lim _{\delta \rightarrow 0} \int_{Q-Q_{\delta}}|c(x)||u(x)|\left|u\left(x^{\delta}(x)\right)\right| \rho(x) d x=0
$$

and this completes the proof.

\section{The energy estimate}

Consider the elliptic equation of the form

(1') $L u+\lambda u=-\sum_{i, j=1}^{n} D_{j}\left(a_{i j}(x) D_{i} u\right)+\sum_{i=1}^{n} b_{i}(x) D_{i} u+(c(x)+\lambda) u=f(x)$

in $Q$, where $\lambda$ is a real parameter.

The results of Section 3 suggest the following concept of the Dirichlet problem.

Let $\phi \in L^{2}(\partial Q)$. A weak solution $u \in W_{1 \text { loc }}^{1,2}(Q)$ of $\left(I^{\prime}\right)$ (or $(1)$ ) is a solution of the Dirichlet problem with the boundary condition

$$
u(x)=\phi(x) \text { on } \partial Q
$$

if

$$
\lim _{\delta \rightarrow 0} \int_{\partial Q}\left[u\left(x_{\delta}(x)\right)-\phi(x)\right]^{2} d S_{x}=0
$$

Under stronger assumptions the above form of the Dirichlet problem has already been considered by Něcas [12], [13] (Chapter 6), Guščin and Mikhailov [3] and Mikhailov [9]. The relation to the Dirichlet problem in $W^{l, 2}(Q)$ will be discussed in the next section. 
We now establish the following energy estimate.

THEOREM 5. Let $u \in W_{l o c}^{l, 2}(Q)$ be a solution of the Dirichlet problem (1'), (13). Then there exist positive constants $d, \lambda_{0}$ and $C$ independent of $u$, such that

$$
\begin{aligned}
\int_{Q}|D u(x)|^{2} r(x) d x+\sup _{0 \leq \delta \leq d} M(\delta)+ & \int_{Q_{d}} u(x)^{2} d x \\
& \leq C\left(\int_{\partial Q} \phi(x)^{2} d S+\int_{Q} f(x)^{2} r(x)^{\theta} d x\right)
\end{aligned}
$$

for $\lambda \geq \lambda_{0}$.

Proof. Note that if $u \in W_{l o c}^{l, 2}(Q)$ is a solution of the Dirichlet problem ( $\left.{ }^{\prime}\right),(13)$, then the conditions I, II, III hold. Let $v$ be the test function introduced in the proof of Theorem 1 . Thus we have

$$
\begin{aligned}
\frac{1}{2} \int_{\partial Q_{\delta}} & \sum_{i, j=1}^{n} a_{i j} D_{i} \rho D j \rho u^{2} \Phi^{2} d S \\
& =\int_{Q_{\delta}} \sum_{i, j=1}^{n} a_{i j} D_{i} u D_{j} u(\rho-\delta) \Phi^{2} d x-\frac{1}{2} \int_{Q_{\delta}} \sum_{i, j=1}^{n} D_{i}\left(a_{i j} D_{j} \rho \Phi^{2}\right) u^{2} d x \\
& +2 \int_{Q_{\delta}} \sum_{i, j=1}^{n} a_{i j}{ }^{D} u \cdot u(\rho-\delta) \Phi D_{j} \Phi d x+\int_{Q_{\delta}} \sum_{i=1}^{n} b_{i} D_{i} u \cdot u(\rho-\delta) \Phi^{2} d x \\
& +\int_{Q_{\delta}}(c+\lambda) u^{2}(\rho-\delta) \Phi^{2} d x-\int_{Q_{\delta}} f u(\rho-\delta) \Phi^{2} d x .
\end{aligned}
$$

Since we may assume that $\delta_{1} \leq 1$, it is clear from the proof of $I \Rightarrow I I$ (Theorem 1) that

$$
\begin{aligned}
\int_{\partial Q_{\delta}} u^{2} d S_{x} \leq L_{1} & {\left[\int_{Q_{\delta}}|D u|^{2}(\rho-\delta) \Phi^{2} d x+\int_{Q} u^{2} \Phi^{2} d x\right.} \\
& +\int_{Q} u^{2}|D \Phi|^{2} d x+\int_{Q_{\delta}} u^{2}(\rho-\delta)|D \Phi|^{2} d x+\lambda \int_{Q_{\delta}} u^{2}(\rho-\delta) \Phi^{2} d x \\
& \left.+\int_{Q_{\delta}} u^{2}(\rho-\delta)^{-\beta_{\Phi}^{2}} d x+\int_{Q_{\delta}} f^{2}(\rho-\delta)^{\theta} \Phi^{2} d x\right]
\end{aligned}
$$


for all $\delta \in\left(0, \delta_{0} / 2\right)$, where $L_{1}$ is a positive constant independent of $\delta, \beta=\min \left(\alpha, 1-2 \varepsilon, 1-2 \varepsilon_{1}\right), \alpha=1-\theta ;$ the constants $0<\varepsilon<1$ and $0<\varepsilon_{1}<1$ were introduced in the proof of $I \Rightarrow I I$ (Theorem 1 ). On the other hand it follows from (15) and assumption (A) that

$$
\begin{aligned}
& \gamma^{-1} \int_{Q_{\delta}}|D u|^{2}(\rho-\delta) \Phi^{2} d x+\lambda \int_{Q_{\delta}} u^{2}(\rho-\delta) \Phi^{2} d x \\
& \leq \int_{\partial Q_{\delta}} \sum_{i, j=1}^{n} a_{i j} D_{i} \rho D_{j} \rho u^{2} \Phi^{2} d S+\frac{1}{2} \int_{Q i, j=1} \sum_{i}^{n} D_{i j}\left(a_{i j j} \rho \Phi^{2}\right) u^{2} d x \\
&-\int_{Q_{\delta}} \sum_{i, j=1}^{n} a_{i j}{ }_{i, j} u \cdot u(\rho-\delta) \Phi D_{j} \Phi d x-\int_{Q_{\delta}} \sum_{i=1}^{n} b_{i} D_{i} u \cdot u(\rho-\delta) \Phi^{2} d x \\
&-\int_{Q_{\delta}} c u^{2}(\rho-\delta) \Phi^{2} d x+\int_{Q_{\delta}} f u(\rho-\delta) \Phi^{2} d x
\end{aligned}
$$

Denote the fourth and fifth integrals on the right hand side of the last inequality by $I_{1}$ and $I_{2}$. As in the proof of Theorem $I$ we have

$$
\begin{aligned}
\left|I_{1}\right| \leq L_{2} \delta{ }_{1}^{\left(1-\varepsilon \delta_{1}\right) / s} 1\left[\int_{Q_{\delta}}|D u|^{2}(\rho-\delta) \Phi^{2} d x+\right. & \int_{Q_{\delta}} u^{2}(\rho-\delta)^{2 \varepsilon-1} \phi^{2} d x \\
& \left.+\int_{Q_{\delta}} u^{2}(\rho-\delta)|D \Phi|^{2} d x\right],
\end{aligned}
$$

(19) $\left|I_{2}\right| \leq L_{3} \delta_{1}^{\left(1-2 \varepsilon_{1} \delta_{1}\right) / r_{1}}\left[\left[\int_{Q_{\delta}}|D u|^{2}(\rho-\delta) \Phi^{2} d x+\int_{Q_{\delta}} u^{2}(\rho-\delta)^{2 \varepsilon_{1}-1} \Phi^{2} d x\right.\right.$

$$
\left.+\int_{Q_{\delta}} u^{2}(\rho-\delta)|D \Phi|^{2} d x\right]
$$

Choose $\delta_{1}$ such that

$$
L_{2} \delta_{1}^{\left(1-2 \varepsilon s_{1}\right) / s_{1}}+L_{3} \delta_{1}^{\left(1-2 \varepsilon_{1} s_{1}\right) / s_{1}} \leq \frac{\gamma^{-1}}{4}
$$

Consequently we deduce from (17), (18) and (19) that 
(20) $\int_{Q_{\delta}}|D u|^{2}(\rho-\delta) \Phi^{2} d x+\lambda \int_{Q_{\delta}} u^{2}(\rho-\delta) \Phi^{2} d x$

$$
\begin{array}{r}
\leq L_{4}\left[M(\delta)+\int_{Q} u^{2} \Phi^{2} d x+\int_{Q_{\delta}} u^{2}(\rho-\delta)|D \Phi|^{2} d x+\int_{Q} u^{2}|D \Phi|^{2} d x\right. \\
\left.+\int_{Q_{\delta}} u^{2}(\rho-\delta)^{-\beta_{\Phi^{2}} d x}+\int_{Q_{\delta}} f^{2}(\rho-\delta)^{\theta} \Phi^{2} d x\right]
\end{array}
$$

for all $\delta \in\left(0, \delta_{1} / 2\right]$, where $L_{4}$ is a positive constant. Letting $\delta$ tend to zero we therefore obtain

$$
\begin{aligned}
\int_{Q}|D u|^{2} \rho(x) \Phi^{2} d x+\lambda \int_{Q} u^{2} \rho^{2} \Phi^{2} d x & \\
\leq I_{4}\left[\int_{\partial Q} \phi(x)^{2} d S+\int_{Q} u^{2} \Phi^{2} d x\right. & +\int_{Q} u^{2} \rho|D \Phi|^{2} d x+\int_{Q} u^{2}|D \Phi|^{2} d x \\
& \left.+\int_{Q} u^{2} \rho^{-\beta} \Phi^{2} d x+\int_{Q} f^{2} \rho^{\theta} \Phi^{2} d x\right]
\end{aligned}
$$

Now let $\Psi$ be a smooth function on $\bar{Q}$ such that $\Psi=1$ on $Q_{\delta_{2}}, \Psi=0$ on $Q-Q_{\delta_{3}}, 0 \leq \Psi \leq 1$ on $\bar{Q}$, where $0<\delta_{3}<\delta_{2} \leq \delta_{0}$. Taking $v=u \Psi^{2}$ as a test function by a standard argument, we deduce that (22) $\int_{Q}|D u|^{2} \Psi^{2} d x+\lambda \int_{Q} u^{2} \Psi^{2} d x$

$$
\leq L_{5}\left[\int_{Q} u^{2} \Psi^{2} d x+\int_{Q} u^{2}|D \Psi|^{2} d x+\int_{Q} f^{2} \Psi^{2} d x\right]
$$

Since

$$
\int_{Q}|D u|^{2} \Psi^{2} d x \geq \int_{Q_{\delta_{2}}}|D u|^{2} d x \geq \frac{1}{\delta^{*}} \int_{Q_{\delta_{2}}}|D u|^{2} \rho(x) d x,
$$

where $\delta^{*}=\sup _{Q} \rho(x)$, by choosing $\delta_{2}=\delta_{1}$ and using (21) and (22) we obtain 
(23)

$$
\begin{aligned}
\int_{Q}|D u|^{2} \rho(x) d x & +\lambda \int_{Q} u^{2} \rho d x \\
\leq L_{6}\left[\int_{\partial Q} \phi(x)^{2} d x\right. & +\int_{Q} u^{2} d x+\int_{Q} u^{2}|D \Psi|^{2} d x+\int_{Q} u^{2}|D \Phi|^{2} d x \\
& \left.+\int_{Q} u^{2} \rho^{-\beta} d x+\int_{Q} f^{2} \rho^{\theta} d x+\int_{Q} f^{2} \Psi^{2} d x\right] .
\end{aligned}
$$

Thus combining (16) and (23) we obtain

(24) $\int_{\partial Q_{\delta}} u^{2} d S$

$$
\begin{aligned}
\leq L_{7}\left[\int_{\partial Q} \phi^{2} d x+\int_{Q} u^{2} d x+\int_{Q} u^{2}|D \Phi|^{2} d x+\int_{Q} u^{2}|D \Psi|^{2} d x\right. \\
\left.\quad+\int_{Q_{\delta}} u^{2}(\rho-\delta)^{-\beta} d x+\int_{Q} u^{2} \rho^{-\beta} d x+\int_{Q} f^{2} \rho d x+\int_{Q} f^{2} \Psi^{2} d x\right]
\end{aligned}
$$

for all $\delta \in\left(0, \delta_{1} / 2\right]$, where $L_{7}$ is a positive constant. To proceed further we derive from Lemma 1 that

(25) $\int_{Q_{\delta}} u^{2}(\rho-\delta)^{-B} d x$

$$
\leq K\left[\delta_{1}^{-\beta} \int_{Q_{\delta_{1}}} u^{2} d x+\delta_{1}^{1-\beta} \int_{\partial Q_{\delta_{1}}} u^{2} d S+\delta_{1}^{1-\beta} \int_{Q_{\delta}-Q_{\delta_{1}}}|D u|^{2}(\rho-\delta) d x\right] .
$$

On the other hand it follows from Theorem 1 in [11], p. 138, that

$$
\int_{\partial Q_{\delta_{1}}} u^{2} d S_{x} \leq \tilde{c}\|u\|_{W^{1}, 2}^{2}\left(Q_{\delta_{1}}\right)
$$

where $\tilde{C}$ is an absolute constant independent of $\delta_{1}$. Combining (22) and (26) we obtain

$$
\int_{\partial Q_{\delta_{1}}} u^{2} d S_{x} \leq \tilde{C}_{1}\left[\int_{Q} u^{2} d x+\int_{Q} u^{2}\left|D_{x} \Psi\right|^{2} d x+\int_{Q} f^{2} \Psi^{2} d x\right] .
$$

Choosing $\delta_{1}$ such that $L_{T} K \delta_{1}^{1-B}<1$, the estimates (23), (24), (25) and (27) imply 


$$
\begin{aligned}
\int_{Q}|D u|^{2} \rho d x+\lambda \int_{Q} u^{2} \rho d x+M(\delta) & \\
\leq L_{8}\left[\int_{\partial Q} \Phi^{2} d x+\int_{Q} u^{2} d x+\int_{Q} u^{2}|D \Phi|^{2} d x+\right. & \int_{Q} u^{2}|D \Psi|^{2} d x+\int_{Q} u^{2} \rho^{-\beta} d x \\
& \left.+\int_{Q} f^{2} \rho^{\theta} d x+\int_{Q} f^{2} \Psi^{2} d x\right]
\end{aligned}
$$

for $\delta \in\left(0, \delta_{1} / 2\right]$, where $L_{8}$ is a positive constant. We can now easily complete the proof of the theorem. Indeed, note that

$$
\int_{Q} u^{2} d x \leq d \sup _{0 \leq \delta \leq d} M(\delta)+\int_{Q_{d}} u^{2} d x,
$$

and

$$
\int_{Q} u^{2} \rho^{-\beta} d x \leq \frac{d^{1-\beta}}{1-\beta} \sup _{0 \leq \delta \leq d} M(\delta)+\int_{Q_{d}} u^{2} \rho^{-\beta} d x
$$

for $d \leq \delta_{0}$. Taking $d$ sufficiently small and $\lambda$ sufficiently large, say $\lambda \geq \lambda_{0}$, the result follows from (28), (29) and (30).

We mention here that Guščin and Mikhailov [3], [9], also proved the energy estimate. The proof presented here is entirely different.

\section{Application to the Dirichlet problem}

In this section we study the solvability of the Dirichlet problem with boundary data in $L^{2}(\partial Q)$. The definition of the Dirichlet problem in $W_{10 c}^{1,2}(Q)$ of $(1 \cdot),(13)$ has already been introduced in the previous section.

Recall that a function $u \in W^{1,2}(Q)$ is a solution of the Dirichlet problem (1), (13) (or (1'), (13)) if $u$ is a weak solution of (1) (or (1')) and $u-\phi \in \mathbb{W}^{1,2}(Q)$.

In this definition it is assumed that boundary data $\phi$ can be extended to an element of $W^{1,2}(Q)$ which is also denoted by $\phi$. As we pointed out in the Introduction this is a restrictive assumption because not every function in $L^{2}(\partial Q)$ is the trace of an element in $W^{1,2}(Q)$. 
Let us introduce the Hilbert space $\tilde{W}^{\mathcal{X}, 2}(Q)$ of all functions $u(x)$ in $W_{10 c}^{1,2}(Q)$ such that

$$
\|u\|_{\tilde{W}^{], 2}(Q)}^{2}=\int_{Q} u(x)^{2} d x+\int_{Q}|D u(x)|^{2} r(x) d x<\infty .
$$

It is evident that

(31) $\|u\|_{W^{J}, 2}^{2} \leq \max (1, d)\left[\int_{Q}|D u(x)|^{2} r(x) d x+\int_{Q_{d}} u(x)^{2} d x+\sup _{0 \leq \delta \leq d} M(\delta)\right]$.

THEOREM 6. Let $\lambda \geq \lambda_{0}$. Then for every $\phi \in L^{2}(\partial Q)$ there is a zonique solution of the Dirichlet problem (1'), (13) in $W_{10 c}^{1,2}(Q)$.

Proof. Let $\left\{\phi_{m}\right\}$ be a sequence of functions in $L^{1}(\partial Q)$ converging in $L^{2}(\partial Q)$ to the function $\phi$.

Define

$$
f_{m}(x)=\left\{\begin{array}{lll}
f(x) & \text { for } & x \in Q_{1 / m}, \\
0 & \text { for } & x \in Q-Q_{1 / m}
\end{array},\right.
$$

for $m$ such that $1 / m \leq \delta_{0}$. Let $u_{m}$ be a solution of the Dirichlet problem

$$
\begin{aligned}
L u+\lambda u & =f_{m} \text { in } Q, \\
u & =\phi_{m} \text { on } \partial Q,
\end{aligned}
$$

in $W^{l, 2}(Q)$ (see [15], [16] or [5]). Here we may assume that $\lambda_{0}$ is sufficiently large that the theorems on the existence of solutions in $W^{l, 2}(Q)$ are applicable. It is obvious that inequality (14) is valid for $u_{m}$ and 


$$
\begin{aligned}
\int_{Q}\left|D u_{p}-D u_{q}\right|^{2} r(x) d x+\sup _{0 \leq \delta \leq d} \int_{\partial Q_{\delta}}\left(u_{p}-u_{q}\right)^{2} d s_{x}+\int_{Q_{d}}\left(u_{p}-u_{q}\right)^{2} d x \\
\quad \leq c\left(\int_{Q}\left(f_{p}-f_{q}\right)^{2}{ }^{\theta} d x+\int_{\partial Q}\left(\phi_{p}-\phi_{q}\right)^{2} d S_{x}\right)
\end{aligned}
$$

It follows from (31) that $\lim _{m \rightarrow \infty} u_{m}=u$ exists in $\tilde{W}^{1,2}(Q)$. As in [9] one can show that $u$ satisfies the boundary condition (13) in $L^{2}(\partial Q)$.

THEOREM 7. Suppose that $\int_{Q} f(x)^{2} r(x)^{2} d x<\infty$ and let $\phi \in L^{2}(\partial Q)$. If there is a function $\phi_{1}$ in $W^{1,2}(Q)$ such that $\phi_{1}=\phi$ on $\partial Q$ (in the sense of trace), then a solution $u(x)$ in $W_{1 \text { loc }}^{1,2}(Q)$ of the Dirichlet problem (1), (13) is a solution in $W^{l, 2}(Q)$ of the same problem.

The proof is essentially the same as that of Lemma 3 in [9] and therefore is omitted.

Theorem 7 implies that every solution in $W_{10 c}^{1,2}(Q)$ of the problem

$$
\left\{\begin{array}{l}
L u=0 \text { on } Q, \\
u=0 \text { in } \partial Q,
\end{array}\right.
$$

or of the problem

$$
\left\{\begin{aligned}
L^{*} \mathcal{U}=0 & \text { on } \partial Q, \\
u=0 & \text { in } \partial Q,
\end{aligned}\right.
$$

is a solution in $W^{1,2}(Q)$. Consequently the problem (32) or $\left(32^{*}\right)$ can have only a finite number of linearly independent solutions (see [5], p. 156, or [15], [16]).

It is known that for the Dirichlet problem (1), (13) to have a solution $W^{l, 2}(Q)$ (we assume that the boundary data is the trace of an element in $W^{l, 2}(Q)$ ), it is necessary and sufficient that $f$ and $\phi$ be related as follows: 


$$
\int_{\partial Q} \frac{\partial u_{j}^{*}}{\partial N} \phi d S_{x}=\int_{Q} u_{j}^{*} f d x \quad(j=1, \ldots, p),
$$

where $\left\{u_{j}^{*}\right\}$ is a maximal linearly independent system of solutions of the problem $\left(32^{*}\right)$ and $\partial / \partial N$ denote the conormal derivative of the elliptic operator (1). If $a_{i j}, b_{i} \in C^{l}(Q)$ then $u_{j}^{*} \in W^{2,2}(Q)$ and Mikhailov [9] was able to show that the Dirichlet problem (1), (13) in $W_{10 c}^{1,2}(Q)$ has a solution if and only if (33) holds. Nevertheless we can show in our situation that the Dirichlet problems in $W^{1,2}(Q)$ and $W_{l o c}^{1,2}(Q)$ have the same eigenvalues.

Fix $\lambda$, and consider the Dirichlet problem

$$
\begin{aligned}
L u+\left(\lambda_{0}+\lambda\right) u & =f \text { on } Q, \\
u & =\phi \text { in } \partial Q,
\end{aligned}
$$

where $\phi \in L^{2}(\partial Q)$ and $\int_{Q} f(x)^{2} r(x)^{2} d x<\infty$.

In view of Theorem 6 the Dirichlet problem (34), (35) has a unique solution $u_{0}$ in $W_{10 c}^{1,2}(Q)$ provided $\lambda_{0}$ is sufficiently large.

THEOREM 8. Let $\phi \in L^{2}(\partial Q), \int_{Q} f(x)^{2} r(x)^{2} d x<\infty$. Then the Dirichlet problem (1'), (13) has a solution in $W_{10 c}^{1,2}(Q)$ if and only if $\lambda_{0} u_{0}$ is orthogonal to every eigenfunction of the problem

$$
L^{*} u+\lambda u=0 \text { in } Q, u=0 \text { on } \partial Q \text {. }
$$

Proof. Suppose that $u$ is a solution of the problem (1'), (13) in $W_{10 c}^{1,2}(Q) \cdot$ Put

$$
w=u-u_{0}
$$

Then $w$ is a solution in $W_{10 c}^{1,2}(Q)$ of the problem

$$
L w+\lambda w=\lambda_{0} u_{0} \text { in } Q, w=0 \text { on } \partial Q
$$


It follows from Theorem 7 that $w \in \mathscr{O}^{l, 2}(Q)$ and consequently $\lambda_{0} u_{0}$ is orthogonal to every eigenfunction of the problem (36).

Conversely if $\lambda_{0} u_{0}$ is orthogonal to every eigenfunction of the problem (36) then the problem $(38)$ has a unique solution in $W^{l, 2}(Q)$ and the solution of the Dirichlet problem (1'), (13) is given by the formula (37).

Observe that the solution of the Dirichlet problem in $W_{10 c}^{l, 2}(Q)$ belongs to $\tilde{W}^{I, 2}(Q)$.

THEOREM 9. Suppose that $f \in L^{q}(Q)$ for some $q>n / 2$ and $c$ is. bounded away from zero on $Q$. Then for every $\phi \in C(\partial Q)$ there exists a unique solution in $\tilde{W}^{1,2}(\bar{Q})$ ก $C(\bar{Q})$ of the Dirichlet problem (1), (13).

Proof. It follows from Theorem 3.8 in [15] that the Dirichlet problem $L u=0$ in $Q, u=0$ on $\partial Q$ has only the trivial solution in $\stackrel{\circ}{W}^{2}(Q)$. Consequently by Theorem 8 there exists a unique solution $u \in \tilde{W}^{l, 2}(Q)$ of the problem (1), (13). It remains only to show that $u \in C(\bar{Q})$. Let $\left\{\phi_{m}\right\}$ be a sequence in $C^{\perp}(\bar{Q})$ converging uniformly to $\phi$ on $\partial Q$. By virtue of Theorem 14.1 in [5] the solution of the Dirichlet problem

$$
\begin{aligned}
L u+\lambda u & =f \text { in } Q, \\
u & =\phi_{m} \text { on } \partial Q,
\end{aligned}
$$

belongs to $W^{1}, 2(Q) \cap C(\bar{Q})$. By Theorem 3.8 in [15],

$$
\sup _{Q}\left|u_{p}(x)-u_{q}(x)\right| \leq \sup _{Q}\left|\phi_{p}(x)-\phi_{q}(x)\right| \rightarrow 0
$$

as $p, q \rightarrow \infty$ and the result follows.

This theorem is similar to, but slightly sharper than, Theorem 8.30 in [2] (p. 196). 


\section{References}

[1] J. Chabrowski and B. Thompson, "On traces of solutions of a semilinear partial differential equation of elliptic type", Ann. Polon. Math. (to appear).

[2] David Gilbarg and Neil S. Trudinger, Elliptic partial differential equations of second order (Die Grundlehren der mathematischen Wissenschaften, 224. Springer-Verlag, Berlin, Heidelberg, New York, 1977.

[3] А.К. Гуцин, В.П. Мххайлов [А.К. Guščin, V.P. Mikaylov], "O Граничных значениях в $L_{p}, p>1$, решений эллиптических уравнений" [Boundary values in $L_{p}(p>1)$ of solutions of elliptic equations ], Mat. Sb. 108 (150) (1979), 3-21.

[4] Alois Kufner, Oldrich John and Svatopluk Fucik, Function spaces (Noordhoff, Leyden; Academia, Prague, 1977).

[5] Olga A. Ladyzhenskaya and Nina N. Ural'tseva, Linear and quasilinear eliiptic equations (Mathematics in Science and Engineering, 46. Academic Press, New York and London, 1968).

[6] J.E. Littlewood and R.E.A.C. Paley, "Theorems on Fourier series and power series (II)", Proc. London Math. Soc. (2) 42 (1937), $52-89$.

[7] В.П. Михайлов [V.P. міkarlov], "О Граничных значениях решений алиптических уравнений второго порядка" [The boundary values of the solutions of second order elliptic equations], Mat. $S b .100$ (142) (1976), 5-13.

[8] В.П. Михайлов [V.P. мікатlov], "О Граничњых значениях решений әллиптически уравнений в областях с глалкой границй" [Boundary values of the solutions of elliptic equations in domains with a smooth boundary], Mat. Sb. 101 (143) (1976), $163-188$.

[9] В.П. Михайлов [V.P. міkaĭlov], "0 задаче дирихле для әлиптического уравнения второго поряпка" [The Dirichlet problem for a second order elliptic equation], Differential'nye Uraumenija 12 (1976), $1877-1891$. 
[10] V.P. Mikhailov, "On the boundary values of the solutions of elliptic equations", Appl. Math. Optim. 6 (1980), 193-199.

[11] В.П. Михайлов [V.P. МіkaY̌lov], Дифференциальные уравнения в частних npouзводныx [Partial differential equations] (Izdat. "Nauka", Moscow, 1976).

[12] Jindřich Nečas, "On the regularity of second-order elliptic partial differential equations with unbounded Dirichlet integral", Arch. Rational Mech. Anal. 9 (1962), 134-144.

[13] Jindřrich Nečas, Les méthodes directes en théorie des équations elliptiques (Masson, Paris; Academia, Prague; 1967).

[14] Friedrich Riesz, "Über die Randwerte einer analytischen Funktion", Math. Z. 18 (1923), 87-95.

[15] Guido Stampacchia, "Le problème de Dirichlet pour les équations elliptiques du second ordre à coefficients discontinus", Ann. Inst. Fourier (Grenoble) 15 (1965), 189-258.

[16] Guido Stampacchia, Equations elliptiques du second ordre a coefficients discontinus (Séminaire de Mathématiques Supérieures, 16; Été, 1965. Les Presses de l'Université de Montréal, Montreal, Quebec, 1966).

Department of Mathematics,

University of Queensland,

St Lucia,

Queensland 4067,

Australia. 